\title{
Clustering and the Synchronization of Oscillator Networks.
}

\author{
Patrick N. McGraw and Michael Menzinger \\ Department of Chemistry, University of Toronto, \\ Toronto, Ontario, Canada M5S $3 H 6$
}

\begin{abstract}
By manipulating the clustering coefficient of a network without changing its degree distribution, we examine the effect of clustering on the synchronization of phase oscillators on networks with Poisson and scale-free degree distributions. For both types of network, increased clustering hinders global synchronization as the network splits into dynamical clusters that oscillate at different frequencies. Surprisingly, in scale-free networks, clustering promotes the synchronization of the most connected nodes (hubs) even though it inhibits global synchronization. As a result, they show an additional, advanced transition instead of a single synchronization threshold. This clusterenhanced synchronization of hubs may be relevant to the brain which is scale-free and highly clustered.
\end{abstract}

PACS numbers: 05.45.Xt, 89.75.Hc 
A key problem in the study of networks is the relation between network structure and function [1] [2]. Among the attributes most frequently used to characterize network structure are degree distribution and clustering coefficient. The latter, defined as the number of triangles divided by the number of connected triples [3] [4], quantifies the tendency of neighbors of a node to be also neighbors of each other. We refer to this property as structural clustering to distinguish it from frequency or dynamical clustering [6] [7] [8].

A recent algorithm[5] allows one to manipulate the clustering coefficient by re-wiring the network, without changing its degree distribution. Kim used this technique to study the effect of clustering on the performance of variously structured Hopfield networks [5], and we use it here to study its effect on synchronization.

In search of factors that control the synchronization of networks of oscillators, researchers have studied the effects of different structural and statistical attributes [6]-[21]. These studies have included systems of coupled maps 6] 86] [9] [10], continuous-time chaotic oscillators [11] [12] 13] [14], spiking neurons [15], and phase oscillators [7] 16] 17] [18]. Unsurprisingly, shortcuts in small-world networks tend to improve synchronization compared to regular lattices 4] 9] 11] [15] 16]. However, other factors such as degree heterogeneity [14], maximum betweenness centrality 19], asymmetry and weighting of couplings [20] [21] also play a role.

In the present work we used Kim's procedure [5] to study the effects of structural clustering on the synchronization of networks of Kuramoto-like[22] phase oscillators with Poisson and scale-free degree distributions. For both network types we found that increased clustering impedes global synchronization and magnifies the fluctuations of the global order parameter. Clustering also qualitatively changes the onset of synchronization. At low clustering [16] [17], the transition is similar to that in the mean-field or globally coupled Kuramoto model, where a single subset of oscillators becomes entrained at a central frequency. With increasing coupling, this synchronized subset entrains larger portions of the remaining oscillators. At higher clustering, on the other hand, synchronization begins with more than one subset, each synchronized at a different frequency, and continues at increased coupling through the recruitment of remaining oscillators by the different subsets. We refer to these subsets as frequency clusters. In contrast to Poisson networks, characterized by a single synchronization transition, we find that strongly clustered scale-free networks show a second synchronization transition which is advanced relative to the principal transition, i.e. it occurs at a lower 
value of the coupling strength. Thus, structural clustering promotes synchronization at low coupling, while inhibiting it at higher coupling. The advanced synchronization begins with the hubs, or highest-degree nodes. These results appear to be relevant to natural networks, such as the brain [23] and ganglia [4] 22] 24] that have higher clustering coefficients than those predicted by simple growth models. In the brain the distribution of functional connections and the probability of finding a link vs. distance are both scale free [23].

In contrast to approaches 11] 12] 14] [19] based on the master stability function 25], we consider non-identical oscillators and their full dynamics, including states where only some of the oscillators are synchronized.

We consider networks of oscillators obeying the coupled differential equations

$$
\frac{d \phi_{i}}{d t}=\omega_{i}+\frac{\lambda}{\langle k\rangle} \sum_{j} a_{i j} \sin \left(\phi_{i}-\phi_{j}\right),
$$

where $0 \leq \phi_{i}<2 \pi$ are $N$ phase variables, $\omega_{i}$ are the randomly and uniformly distributed intrinsic frequencies, $\lambda$ is the coupling strength, and $a_{i j}$ is the adjacency matrix ( 1 if $i$ and $j$ are connected, 0 otherwise). The coupling strength is scaled by the average degree $\langle k\rangle$ of the nodes, averaged over the whole network. In the globally coupled Kuramoto model [22] where $a_{i j}=1, \forall i \neq j$, this reduces to scaling by the number of oscillators $N$. The above form makes the coupling strengths symmetric and weights all links equally [17], but in the case of a nonhomogeneous degree distribution, some oscillators may receive a stronger synchronizing signal because they have more neighbors.

Results are for networks with $N=1000$ nodes and average degree $\langle k\rangle \approx 20$. Qualitatively similar results were obtained with $N=5000$ and $\langle k\rangle=6$. We consider a random (Poisson) network and a scale-free network generated by the Barabasi-Albert preferential attachment algorithm [26], and families of networks derived from each of these by changing the clustering coefficient $\gamma$. To vary $\gamma$, we use a stochastic rewiring algorithm [5] that rearranges connections with a bias toward increased clustering. The procedure is as follows: 1) Pick randomly two existing links. 2) Compute whether interchanging these links increases or decreases the total number of triangles. Perform the interchange only if it increases that number. 3) Repeat these steps until the desired clustering is achieved. (One can reduce the clustering coefficient by reversing the acceptance criterion). Since this algorithm only rewires connections and does not change the degree of any node, the degree distribution as well as the degree sequence is fixed. 
The random $\omega_{i}$ values were uniform distributed over the interval $0.9 \leq \omega_{i} \leq 1.1$ and the initial phases were also random. We ran the dynamics at a series of increasing values of $\lambda$, integrating the equations using a simple Euler method with step size 0.02. After 100 time units to allow for relaxation to a steady state, we measured the time-averaged (over 500 time units) synchronization order parameter [22]

$$
m \equiv\left\{\left|\frac{1}{N} \sum_{j=1}^{N} e^{i \phi_{j}}\right|\right\} .
$$

$m$ is of order $1 / \sqrt{N}$ if the oscillators are uncorrelated and approaches 1 when all are in phase. In addition, we measured the frequency $\Omega_{i} \equiv\left\{\dot{\phi}_{i}\right\}$ of each oscillator over the same 500-unit time interval after relaxation. The brackets \{\} signify time averaging. This measurement reveals the collective behavior in detail.

To study the effect of clustering on the transition to synchronization, we first examine the order parameter $m$ as a function of the coupling strength $\lambda$. Figure 1 shows plots of $m$ vs. $\lambda$ for networks with Poisson and scale-free degree distributions at several values of the clustering coefficient $\gamma$. In each plot the data were averaged over time, over several realizations of the intrinsic frequencies and over several network rearrangements. The degree sequence, however, was the same in all cases. Poisson networks (fig.1A) behave more simply than scale-free ones. The natural (low-clustering) random network shows a rapid transition to order above $\lambda=0.12$. Increasing clustering inhibits global synchronization and makes this transition less steep. The lowered mean values of $m$ in the highly clustered networks are associated with extremely large temporal fluctuations around the mean.

In scale-free networks (fig.1B,C), clustering has a more complicated effect. The network with $\gamma=0$ shows the onset of synchronization (upward turn in the graph) near $\lambda \approx 0.07$ and the transition to full synchronization is not as steep as in the Poisson case. Surprisingly, increased clustering slightly enhances the order parameter at weak coupling but suppresses it at strong coupling. For intermediate $\gamma$ this results in two separate transitions, an advanced transition (threshold at $\lambda \approx 0.03$ ) to partial synchronization followed by a delayed one $(\lambda \approx 0.15)$ to full synchronization.

For more insight into the synchronization transitions we examine scatter plots of observed frequency $\Omega_{i}$ vs. intrinsic frequency $\omega_{i}$. Each plot in figures 24 shows the population's behavior for one realization of the random variables. In the absence of interactions, all points would fall along the line $\Omega_{i}=\omega_{i}$. In the globally coupled (mean-field) case 22], the 

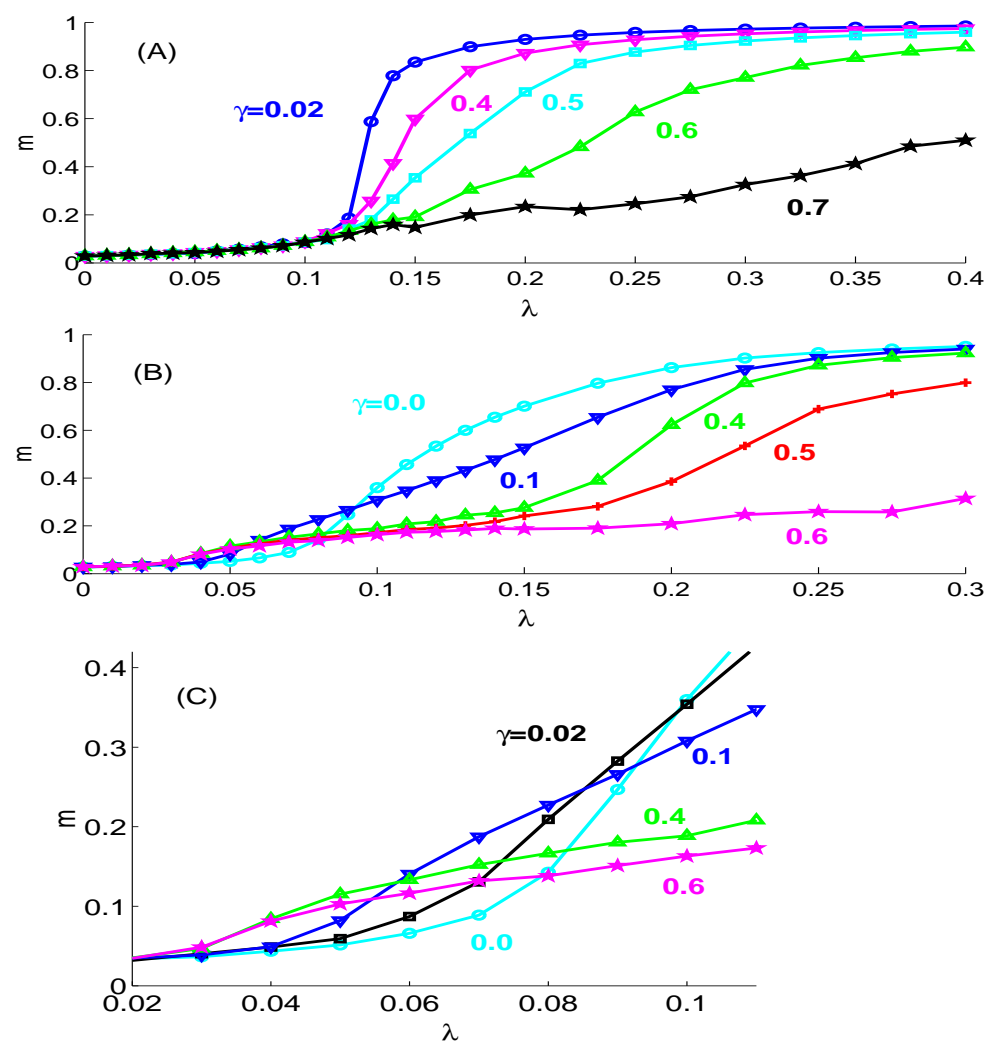

FIG. 1: (color online) Order parameter $m$ vs. coupling strength $\lambda$, for different values of the clustering coefficient $\gamma$. (A) Poisson degree distribution. (B,C) Scale-free degree distribution. (C) Close-up of the transition region showing that increased clustering leads to an advanced (lowerג) transition.

nodes differ only by their intrinsic fequencies. Therefore, the observed frequency is in every case a single-valued function $\Omega(\omega)$ of the intrinsic frequency, whose shape changes with $\lambda$. In particular, the curves begin to flatten around $\omega \approx 1$, as oscillators with frequencies closest to the average are the first to synchronize. The flat portion broadens with increasing coupling. For Poisson networks (fig. 2) at low clustering, the same qualitative picture holds as in the mean-field case except that there is more scatter and the curve is blurred. This is due to the fact that each oscillator's behavior now depends not only on its intrinsic frequency and the global average, but also on the details of its local neighborhood. As in the mean-field case, the oscillators near the extremes of the $\omega$ distribution are the last to become fully entrained. Unlike the mean-field case, some remain unentrained even when the synchronized group is well-established and has recruited members from the entire intrinsic frequency range. At 

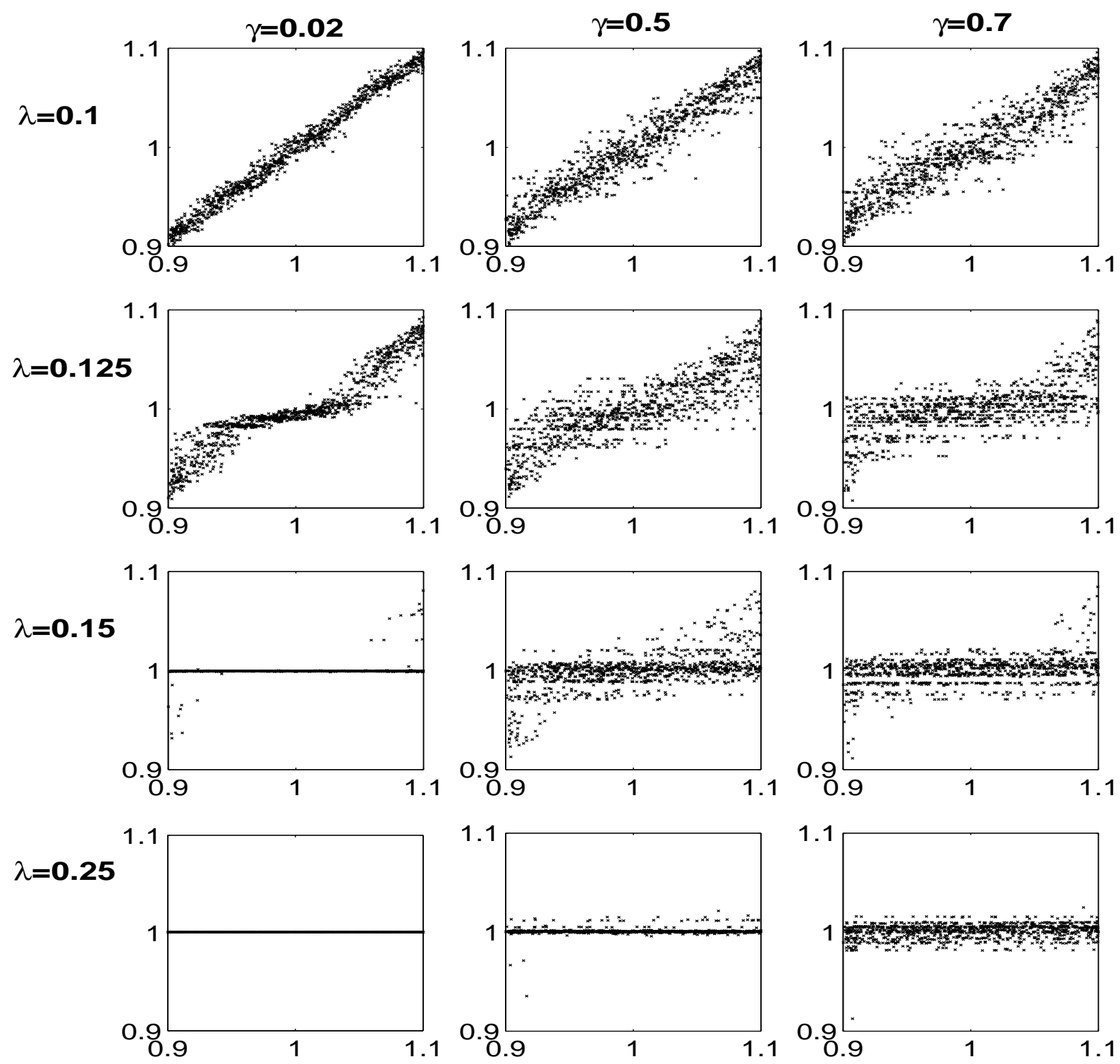

FIG. 2: Poisson networks: Scatter plots of average oscillation frequency $\Omega$ (vertical axis) vs. intrinsic frequency $\omega$ (horizontal). At zero coupling, the points all lie on the line $\Omega=\omega$. With increasing coupling, more oscillators line up at synchronized frequencies. For high clustering, several bands form at different frequencies, rather than one frequency as in the low- $\gamma$ case.

higher clustering, the scatter plots differ even more from the mean-field case, and they do so in two ways. First, the scatter is more pronounced. Second, the horizontal striations indicate that the oscillators cluster in subgroups (frequency clusters) oscillating at different frequencies. As $\lambda$ increases, the frequency clusters converge and eventually merge, but full synchronization requires a stronger coupling compared to networks with low clustering. The 

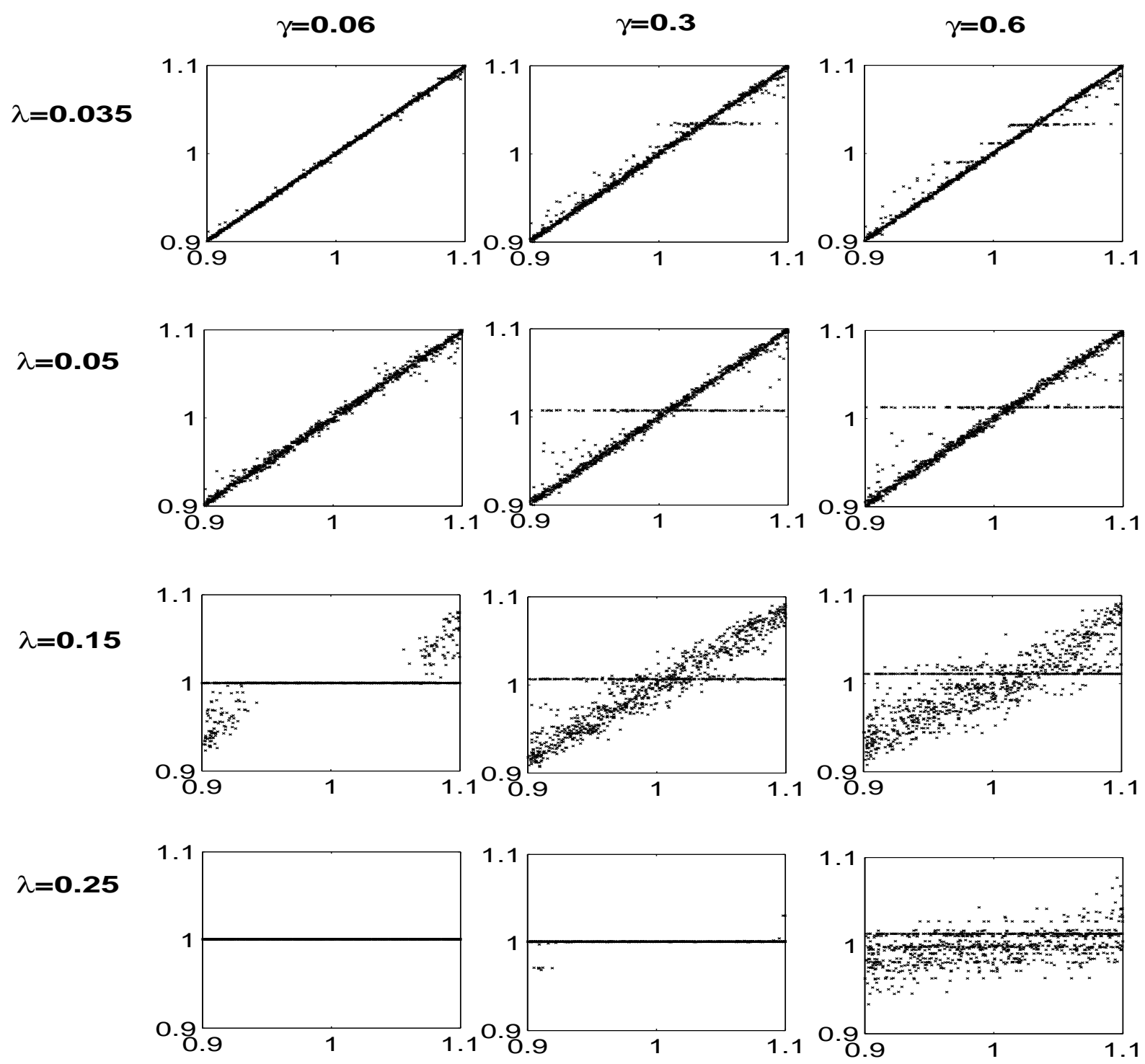

FIG. 3: Scale-free networks: Scatter plots of $\Omega$ vs. $\omega$. Synchronized subsets (horizontal bands) begin to form at weaker coupling for the more clustered networks. Frequency clustering is evident at high $\gamma$.

beating of the different frequencies accounts for the low value of the global order parameter and its large fluctuations.

In the scale-free case, the scatter plots (figure 31) confirm that the highly clustered networks begin to synchronize at a weaker coupling than the less clustered ones. Initially, however, this synchronization only affects a subset of the nodes, while the remaining nodes still fall close to the line $\Omega=\omega$ as if they were not interacting at all. This is in contrast 
$\gamma=0.06$
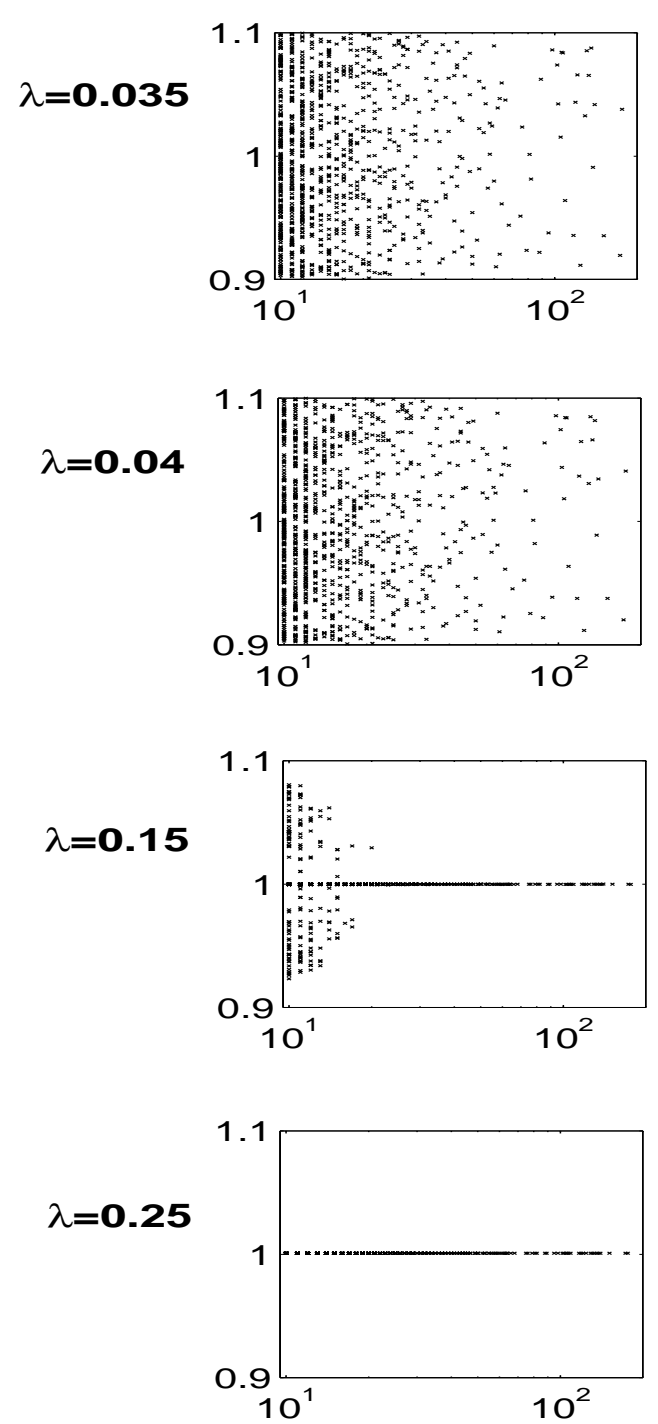

$\gamma=0.3$
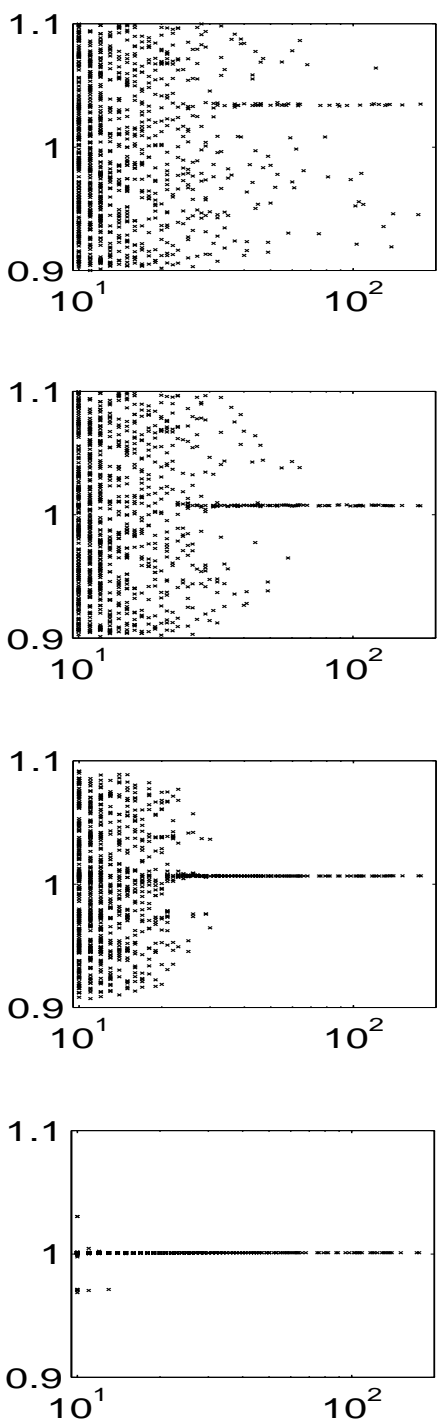

$\gamma=0.6$
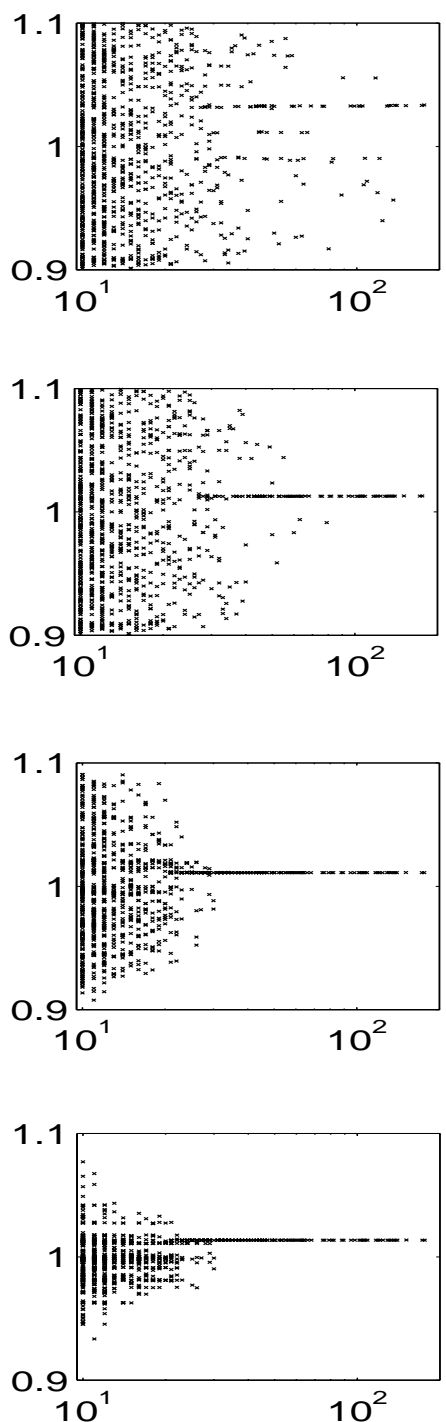

FIG. 4: Scale-free networks: Average frequency $\Omega$ vs degree $k$ at several values of clustering $\gamma$ and coupling strength $\lambda$. The degree (horizontal axis) is plotted on a logarithmic scale. Synchronization begins with the hubs (nodes with the highest $k$ ) and progresses downward to those with lower $k$.

to the Poisson scatter plots which begin to flatten near the center of the frequency range as the transition begins. Since scale-free networks have a strongly heterogeneous degree distribution, the entrainment of an oscillator depends strongly on the number of its inputs. When $\Omega$ is plotted against the degree $k$ of each node as in figure 4, it is apparent that the higher-degree nodes (hubs) begin to synchronize first, while the lower-degree nodes synchronize as the coupling continues to increase. One similarity with the Poisson case 
is that structural clustering enhances frequency clustering. While clustering promotes the formation of synchronized frequency clusters among the hubs, it inhibits the synchronization of the network as a whole. The early hub synchronization accounts for the slightly enhanced order parameter at weak coupling.

In conclusion, we examined the synchronization of networks of non-identical coupled phase oscillators with both Poisson and scale-free degree distributions, and studied the effects of varying the clustering coefficient without affecting the degree distribution. Our first main result, for both types of networks, is that clustering encourages the formation of sub-populations synchronized at different frequencies (frequency clusters), and thus discourages full global synchronization at a single frequency. Our second key finding concerns the scale-free case, where we found that, despite the increased difficulty of full synchronization, higher clustering actually promotes the onset of partial synchronization of the hubs. Scale-free networks with high clustering thus appear to undergo two separate transitions as the coupling strength increases: an early transition to partial synchronization and a delayed one to full synchronization. The first transition involves only the hubs while leaving the majority almost unaffected. The hubs seem to form the growth nuclei for the synchronized state. Interestingly, they are able to synchronize even though many of their inputs come from lower degree nodes which are not yet synchronized. It is known[17] that in ordinary, low-clustered scale-free networks the relaxation time for synchronization of hubs is shorter than that of less connected nodes, but this does not account for the effect of structural clustering in promoting their synchronization. It is possible that increasing clustering may, as a by-product, increase the assortativity of degree mixing [29], so that hubs become more likely to connect to each other. In other models of clustered networks it was found that triangles preferentially include higher-degree nodes[27]. Clustering may also affect the betweenness centrality distributions.

This advanced transition may play a role in highly clustered networks, for instance in the human brain. In natural as opposed to abstract networks, the cost associated with longrange connections often gives them a tendency toward clustering. For the ganglion of $C$. elegans [24], the clustering coefficient is $\gamma=0.28$. For the human brain [23], the connectivity is essentially scale-free in different regions, and clustering lies three orders of magnitude above that of equivalent random networks. This and the weak synaptic coupling strength in the brain [28] 30] point to the potential importance of cluster-enhanced synchronization 
of oscillating hubs.

[1] S.H. Strogatz, Nature 410, 268 (2001).

[2] R. Albert and A.L. Barabasi, Rev. Mod. Phys. 74, 47 (2002); M.E.J. Newman, preprint cond-mat/0202208 (2002); A.L. Barabasi, Linked: The New Science of Networks (Perseus Publishing, Cambridge, MA, 2002).

[3] D.J. Watts and S.H. Strogatz, Nature 393, 440 (1998).

[4] D.J. Watts, Small Worlds (Princeton University Press, Princeton, NJ, 1999). Six Degrees (Norton, New, York, 2003).

[5] B.J. Kim, Phys. Rev. E, 69, 045101(R) (2004).

[6] S.C. Manrubia and A.S. Mikhailov, Phys. Rev. E 60, 1579 (1999); S.C. Manrubia, A.S. Mikhailov, D.H. Zanette, Emergence of dynamical order (World Scientific, Singapore, 2004).

[7] D.H. Zanette and A.S. Mikhailov, Europhysics Letters 65, 465 (2004); Physica D 194, 203 (2004).

[8] S. Jalan and R.E. Amritkar, Phys. Rev. Lett. 90, 014101 (2003); preprint nlin.CD/0307029 (2003); Physica A 321, 220 (2003); 346, 13 (2005).

[9] P.M. Gade and C.-K. Hu, Phys. Rev. E 62, 6409 (2000).

[10] J. Jost and M.P. Joy, Phys. Rev. E 65, 016201 (2001).

[11] M. Barahona and L.M. Pecora, Phys. Rev. Lett 89, 054101 (2002).

[12] X.F. Wang and G. Chen, IEEE Trans. Circuits and Systems I- Fundamental Theory and Applications 49, 54 (2002).

[13] X.F. Wang and G. Chen, Int. J. Bifurcation and Chaos 12, 187 (2002).

[14] T. Nishikawa, A.E. Motter, Y.C. Lai, F.C. Hoppensteadt, Phys. Rev. Lett. 91, 014101 (2003).

[15] L.F. Lago-Fernandez, R. Huerta, F. Corbacho and J.A. Siguenza, Phys. Rev. Lett. 84, 2758 (2000).

[16] H. Hong, M.Y. Choi and B. J. Kim, Phys. Rev. E 65, 026139 (2002).

[17] Y. Moreno and A.F. Pacheco, Europhys. Lett. 68, 603 (2004).

[18] G. Katriel, preprint nlin.PS/0308031 (2003).

[19] H. Hong, B.J. Kim, M.Y. Choi, H. Park, Phys. Rev. E 69, 067105 (2004). 
[20] A.E. Motter, C. Zhou, J. Kurths, cond-mat/0406207 (2004).

[21] D.-U. Hwang, M. Chavez, A. Amann, S. Boccaletti, cond-mat/0412728 (2004).

[22] Y. Kuramoto, Chemical oscillations, waves and turbulence (Springer Verlag, Berlin; 1984) ; A. Pikovsky, Synchronization, (Cambridge University Press, Cambridge; 2001).

[23] V.M. Eguiluz, G. Cecchi, D.R. Chialvo, M. Baliki, A.V. Apkarian, Phys. Rev. Lett. 94, 018102 (2005).

[24] C. Cherniak, J. Neurosci. 14, 2418 (1994).

[25] L. M. Pecora and T.L. Carroll, Phys. Rev. Lett. 80, 2109 (1998).

[26] A.L. Barabasi and R. Albert, Science 286, 509 (1999).

[27] D. Sergi, preprint cond-mat/0412472 (2004).

[28] M.Abeles, Neural Codes for Higher Brain Functions. In: H.J. Markovich (ed.) Information Processing in the Brain.(Hans Huber Publishers, Toronto, 1988).

[29] M.E.J. Newman, Phys. Rev. Lett 89, 208701 (2002).

[30] F.C. Hoppensteadt, E.M. Izhikevich, Weakly Connected Neural Networks. (Springer Verlag, Berlin, 1997). 